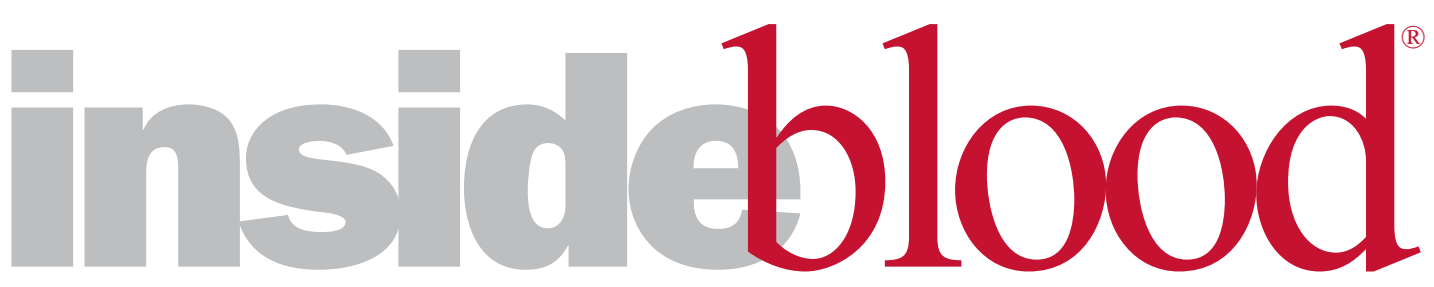

5 SEPTEMBER 2013 | VOLUME 122, NUMBER 10

\section{Old wine in a new bottle: ready to drink?}

Jan J. Cornelissen ${ }^{1}{ }^{1}$ ERASMUS UNIVERSITY MEDICAL CENTER

In this issue of Blood, Walter et al describe the impact of minimal residual disease (MRD) as measured by multicolor flow cytometry (MCF) on relapse, progression-free survival (PFS), and overall survival (OS) in acute myeloid leukemia (AML) patients who received an allogeneic hematopoietic stem cell transplantation (alloHSCT). ${ }^{1}$ ince the concept of MRD was - introduced in the early 1980s, it has only been gradually introduced into clinical practice. $^{2}$ The most important examples are the current standard use of quantitative polymerase chain reaction (qPCR) in patients with chronic myeloid leukemia ${ }^{3}$ and the use of flow cytometry and qPCR in acute lymphoblastic leukemia. ${ }^{4}$ Apart from the use of PCR in acute promyelocytic leukemia, $\mathrm{MRD}$ is currently not routinely monitored in
AML patients. Walter et al report that MRD strongly impacts on PFS and OS in alloHSCT recipients with AML, and in a similar degree in first and second remission. ${ }^{1}$ That effect became evident as from a cutoff level of $0.1 \%$, without significantly changing in patients with increasing levels of MRD.

Although MRD as an innovative concept was first suggested in AML, it took almost 3 decades for MRD to mature in AML. ${ }^{5}$ An

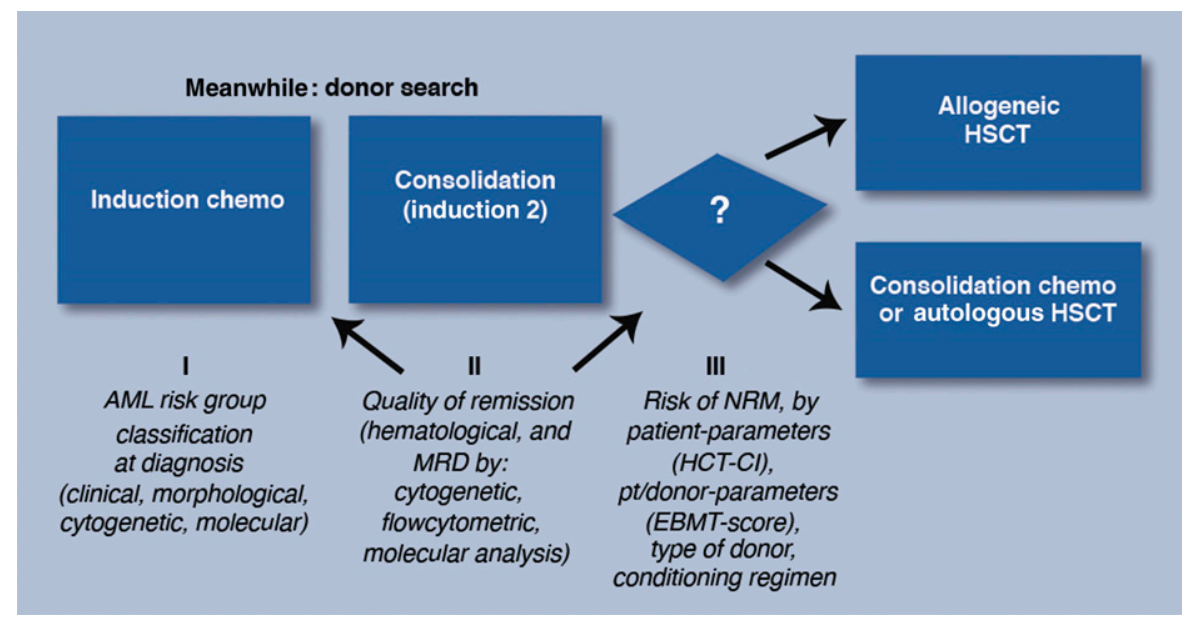

Time frame and parameters for decision making prior to allogeneic hematopoietic stem cell transplantation (HSCT) in AML. HCT-Cl, hematopoietic stem cell transplantation comorbidity index; EBMT, European Group for Blood and Marrow Transplantation. Professional illustration by Marie Dauenheimer. important question to be addressed today is whether recent studies, including the present study from Seattle, urge us to adapt our transplant policy. The choice of postremission treatment in AML is currently under intense debate (see figure). Although alloHSCT provides the strongest antileukemic treatment, the benefit can be compromised by nonrelapse mortality (NRM). Therefore, a careful assessment of the most important variables affecting relapse and NRM should be included in the workup of transplant recipients nowadays. A recent review by the European Leukemia Net (ELN) AML working group ${ }^{6}$ described that approach in detail for AML transplant recipients, taking into account on one hand the parameters predicting relapse and on the other hand parameters predicting NRM in a timedependent fashion (see figure). The ELN recommended to aim for a disease-free survival (DFS) benefit by alloHSCT of at least $10 \%$ for the individual patient as compared with a nonalloHSCT approach. Such a DFS benefit may be achieved in AML intermediate- and poorrisk patients, provided that the risk of NRM does not exceed $25 \%$ and $35 \%$, respectively. Good-risk AML patients, as defined by cytogenetic and molecular hallmarks, ${ }^{6}$ do not currently receive an alloHSCT as part of firstline treatment because the DFS benefit is limited and those patients may effectively be rescued upon relapse. ${ }^{7}$

Should intermediate-risk patients without MRD after induction chemotherapy be considered as good-risk patients? The study by Walter et $\mathrm{al}^{1}$ and by other investigators 8,9 may seem to suggest this, but more information is needed before adapting a transplant policy. First, prospective studies reporting outcome of AML intermediate-risk MRD-negative patients who do not proceed to alloHSCT because of MRD negativity would be needed. Prospective studies, addressing this particular issue have not been reported yet, and the question whether 
alloHSCT can be withheld in that subgroup is still open but urgent. Especially because of a lower rescue rate with reinduction chemotherapy in intermediate-risk patients in general. ${ }^{10}$ Second, methods of MCF vary among different studies. Some of the methods apply MRD monitoring by first establishing a leukemia-associated phenotype (LAP) at diagnosis. ${ }^{8,9}$ The present study applied a different approach by defining MRD by any deviation of the normal pattern of regenerating or normal marrow, which circumvents the need for a LAP and also circumvents the problem of a phenotypic shift that may occur during chemotherapy. This approach may seem very attractive, but from a technical point of view, the method is challenging and so far is only being used by a very limited number of centers. Therefore, the MCF method applied in the present study would need validation in prospective studies on a broader scale. Moreover, such confirmation is also needed to be able to quantitatively weigh all relevant prognostic markers prior to alloHSCT within a single, large study, including parameters predicting for NRM.

MRD-positivity in the present study appeared to be associated with a 3 -year OS of $32 \%$ and $44 \%$ in CR1 and CR2 patients, respectively. Although the relapse rate after alloHSCT was relatively high in those patients, the allogeneic graft-vs-leukemia (GVL) effect was suggested not to differ between MRDnegative and MRD-positive patients. Of interest, as a reflection of GVL activity, the present study showed a strong reduction of relapse (hazard ratio: $0.46(0.24-0.88) ; P=.02)$ in patients with chronic graft-vs-host disease, which was similarly exerted in patients with or without MRD. Therefore, the higher relapse rate in MRD-positive patients does not indicate that GVL was absent, but rather suggests that the allogeneic GVL effect would need to be exploited more effectively. Several approaches may be developed into that direction, but monitoring of MRD at regular intervals after alloHSCT with subsequent adaptation of the immunosuppressive regimen and/or donor lymphocyte infusion may be one of them. In addition, intermediate-risk patients who are MRD-positive after induction and consolidation chemotherapy may be candidates for an alloHSCT using stem cells from alternative donors, which type of transplants is currently not routinely offered to those patients if MRD information is lacking.
Collectively, the present study ${ }^{1}$ and other studies in $\mathrm{AML}^{8,9}$ strongly suggest that the net effect of the combination of an anthracycline and cytarabine in AML currently needs to be evaluated by both flow cytometry and microscopic evaluation to obtain optimal information regarding the risk of relapse. A change of transplant policy seems premature in intermediate-risk patients who become MRD-negative after induction chemotherapy and further prospective studies would be needed. Finally, despite the adverse prognostic impact of MRD-positivity, these patients do benefit from alloHSCT, which, therefore, should be pursued, even with alternative donors, and preferably be optimized by exploiting GVL more effectively. Therefore, after 30 years of MRD maturation in AML, we may now begin to sip of this extraordinary wine.

Conflict-of-interest disclosure: The author declares no competing financial interests.

\section{REFERENCES}

1. Walter RB, Buckley SA, Pagel JM, et al. Significance of minimal residual disease before myeloablative allogeneic hematopoietic cell transplantation for AML in first and second complete remission. Blood. 2013;122(10):1813-1821.

2. Hagenbeek A, Martens AC. Detection of minimal residual disease in acute leukemia: possibilities and

limitations. Eur 7 Cancer Clin Oncol. 1985;21(3):389-395.
3. Hughes TP, Kaeda J, Branford S, et al; International Randomised Study of Interferon versus STI571 (IRIS) Study Group. Frequency of major molecular responses to imatinib or interferon alfa plus cytarabine in newly diagnosed chronic myeloid leukemia. $N$ Engl 7 Med. 2003 ; 349(15):1423-1432

4. van Dongen JJ, Seriu T, Panzer-Grümayer ER, et al. Prognostic value of minimal residual disease in acute lymphoblastic leukaemia in childhood. Lancet. 1998; 352(9142):1731-1738

5. Schuurhuis GJ, Ossenkoppele G. Minimal residual disease in acute myeloid leukemia: already predicting a safe haven? Expert Rev Hematol. 2010;3(1):1-5.

6. Cornelissen JJ, Gratwohl A, Schlenk RF, et al. The European LeukemiaNet AML Working Party consensus statement on allogeneic HSCT for patients with AML in remission: an integrated-risk adapted approach. Nat Rev Clin Oncol. 2012;9(10):579-590.

7. Jourdan E, Boissel N, Chevret S, et al; French AMI Intergroup. Prospective evaluation of gene mutations and minimal residual disease in patients with core binding factor acute myeloid leukemia. Blood. 2013;121(12): 2213-2223.

8. Maurillo L, Buccisano F, Del Principe MI, et al. Toward optimization of postremission therapy for residual disease-positive patients with acute myeloid leukemia. 7 Clin Oncol. 2008;26(30):4944-4951.

9. Terwijn M, van Putten WLJ, Kelder A, et al. High prognostic impact of flow cytometric minimal residual disease detection in acute myeloid leukemia: data from the HOVON/SAKK AML 42A study. 7 Clin Oncol. 2013. In press.

10. Forman SJ, Rowe JM. The myth of the second remission of acute leukemia in the adult. Blood. 2013; 121(7):1077-1082.

C 2013 by The American Society of Hematology

\section{- TRANSPLANTATION}

Comment on Dong et al, page 1802

\section{Tregs, HSCT, and acute GVHD: up close and personal}

John Koreth ${ }^{1}$ and Jerome Ritz ${ }^{1}{ }^{1}$ DANA-FARBER CANCER INSTITUTE

In this issue of Blood, Dong et al present a unique detailed view of human regulatory T-cell (Treg) diversity in homeostatic and pathological states after allogeneic hematopoietic stem cell transplantation (alloHSCT). ${ }^{1}$

A lloHSCT provides curative graft-versustumor potential for patients with hematologic malignancies. However, donor effector immune responses to allogeneic (donor/recipient polymorphic) and autologous (donor/recipient nonpolymorphic) antigens also underlie acute and chronic graft-versus-host disease (GVHD), the major toxicities of this therapeutic approach.

$\mathrm{CD}^{+}{ }^{+} \mathrm{CD} 25^{+}$Foxp $3^{+}$regulatory T lymphocytes comprise $\sim 5 \%$ to $10 \%$ of circulating $\mathrm{CD}^{+}{ }^{+} \mathrm{T}$ cells and migrate to inflammatory sites to control innate and adaptive immune responses, especially those due to effector lymphocytes of helper $\mathrm{T}$ ( $\mathrm{Th}$ ) subsets: Th1, Th2, Th17, and follicular Th cells (reviewed by Ohkura et $\mathrm{al}^{2}$ ). Tregs play a critical role in the prevention of autoimmunity and several studies have suggested that Tregs also play a central role in the establishment and maintenance of immune tolerance after alloHSCT. 


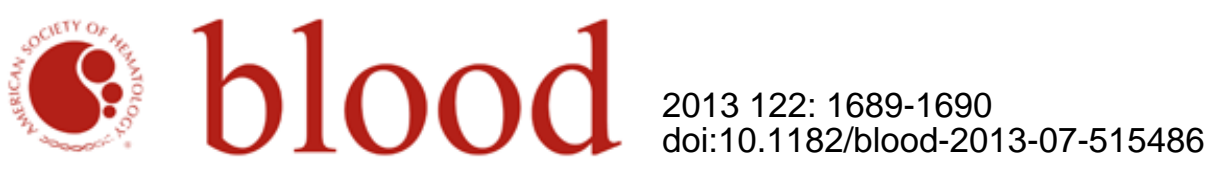

\section{Old wine in a new bottle: ready to drink?}

Jan J. Cornelissen

Updated information and services can be found at: http://www.bloodjournal.org/content/122/10/1689.full.html

Articles on similar topics can be found in the following Blood collections

Free Research Articles (3286 articles)

Information about reproducing this article in parts or in its entirety may be found online at: http://www.bloodjournal.org/site/misc/rights.xhtml\#repub_requests

Information about ordering reprints may be found online at: http://www.bloodjournal.org/site/misc/rights.xhtml\#reprints

Information about subscriptions and ASH membership may be found online at: http://www.bloodjournal.org/site/subscriptions/index.xhtml 